\title{
Gestão de Projetos de Inovação Tecnológica: uma Nova Abordagem para Aumentar a Participação dos Stakeholders
}

\section{Introdução}

Dentre as estratégias adotadas por empresas para aumento de competitividade, a inovação de produtos e processos possibilita a expansão e a criação de novos mercados, incorporando diferenciais competitivos que agregam valor aos seus produtos. Segundo a Pesquisa de Inovação PINTEC 2011, entre 2009 e 2011, aproximadamente R $\$ 85$ bilhões foram investidos em atividades de pesquisa, desenvolvimento e inovação (PD\&I) (IBGE, 2013).

Nas linhas de fomento à inovação existentes no Brasil (e em grande parte do mundo), o uso de Editais públicos é tido como o principal mecanismo de aporte de recursos. Por este instrumento, organizações submetem seus projetos de inovação, os quais são avaliados e, se aprovados, recebem recursos para o desenvolvimento proposto (UNESCO, 2011).

Devido ao grande volume de recursos e a quantidade de projetos em desenvolvimento, as organizações de fomento possuem o desafio de acompanhar o desempenho destes iniciativas. Por este acompanhamento, avaliamse as ações a serem tomadas no portfólio, seja para divulgação dos casos de sucesso ou descontinuidade de projetos sem perspectiva de sucesso.

Tradicionalmente, a gestão da carteira de projetos é realizada por sistemas de gestão baseados em práticas do guia A Guide to the Project Management Body of Knowledge - PMBOK® Guide. Tais práticas, apesar de oferecerem um conjunto detalhado de informações para gestão de projetos, não apresentam um método rápido e de fácil visualização para monitoramento de uma carteira de projetos.
Além disso, dentro da perspectiva do gestor do projeto, não existem quaisquer estímulos ou incentivos para que as ações previstas no projeto sejam realizadas e que as informações presentes nos sistemas de gestão de projetos estejam sempre atualizadas. Como consequência, estes projetos possuem uma alta taxa de inadimplência em sua execução (Zichermann \& Cunningham, 2011).

Diante deste cenário, este artigo tem como objetivo apresentar diferentes métodos para gestão de projetos de inovação tecnológica, visando a um aumento na participação das partes envolvidas no projeto (stakeholders), contribuindo, assim, com o aumento do desempenho na execução e posterior finalização desse.

\section{Metodologia}

A avaliação do desempenho de projetos possui um grande destaque no guia $A$ Guide to the Project Management Body of Knowledge - PMBOK® Guide. Referência em gestão de projetos, o Guia PMBOK®, elaborado pelo Project Management Institute (PMI), fornece diretrizes para o gerenciamento de projetos, definindo conceitos de gestão de cada etapa do ciclo de vida de um projeto (PMBOK Guide, 2013).

Para contemplar todo ciclo de vida de um projeto, o Guia estabelece 47 processos de gestão divididos em grupos de gerenciamento de projetos, dentre os quais, o Grupo de processos de monitoramento e controle estabelece "os processos necessários para acompanhar, revisar e regular o progresso e o desempenho de um projeto, bem como identificar todas as áreas nas quais serão necessárias mudanças no plano e iniciar as 
mudanças correspondentes"(PMBOK GUIDE, 2013). O grupo de processos de monitoramento e controle é dividido em sete processos: escopo, cronograma, custo, qualidade, comunicação, aquisições e risco.

Entretanto, projetos de inovação tecnológica possuem particularidades que vão além de tais processos, uma vez que esses também preconizam a atração de capital externo, seja de organizações de fomento ou de venture capital. Segundo pesquisa realizada por MASON \& STARK (2004), investidores avaliam nove critérios para seleção de projetos a investir: Perfil de Empreendedorismo/Gestão da Equipe, Estratégia, Operações (aspectos práticos da funcionalidade do negócio), Produto/Serviço, Mercado, Considerações financeiras, Adequação do Investidor e Plano de Negócio.

Diferente da abordagem tradicional de identificar métricas independentes para as fases do ciclo de vida de um projeto, PILLAI et al. (2002) propõe um método que realiza a medição dos principais indicadores que os stakeholders consideram importantes em um projeto. Para quantificação, é proposta uma modelagem matemática e, por fim, é calculado um índice de desempenho global do projeto.

Para PILLAI et al. (2002), o sistema de gestão do desempenho do projeto deve ser capaz de fornecer uma medida quantitativa que reflita os seguintes requisitos:

Necessidades e expectativas dos stakeholders;

Atualização contínua das premissas adotadas no passado, devido ao ganho de conhecimento durante a execução do projeto;

Previsão de sucesso ou falha do projeto de forma antecipada, otimizando a utilização de recursos.

Para o alcance destes requisitos, é proposto um método de gestão que monitore as três fases de um projeto: seleção, desenvolvimento e implementação do projeto. Em seguida, são definidos os indicadores de desempenho do projeto pelos stakeholders. Tais indicadores devem possuir características quantificáveis, uma vez que, ao final do processo, calcula-se um índice de desempenho global do projeto.

Com o objetivo de aumentar a participação das partes envolvidas na execução do projeto, foi realizado um estudo de caso, utilizando o método proposto por PILLAI et al. (2002) em uma amostragem de projetos do Edital SENAI SESI de Inovação.

\section{Estudos de Caso}

Como forma de se avaliar os diferentes métodos de gestão de projetos de inovação tecnológica, foi realizado um estudo de caso, envolvendo 10 projetos da edição 2012 do Edital SENAI SESI de Inovação. Neste estudo de caso, foi aplicado um método simplificado de gestão de projetos orientado pelos stakeholders, baseado na proposta de PILLAI et al. (2002) . Para realização deste estudo, foram selecionados projetos de diferentes setores industriais com empresas de diferentes portes. Com esta diversificação, verificou-se que o método permite a comparação do desempenho de projetos de áreas distintas.

A primeira etapa de aplicação do método referese à escolha dos principais stakeholders da carteira de projetos. No caso do Edital SENAI SESI de Inovação são: os representantes da empresa participante, a equipe de especialistas do SENAI ou do SESI e a comissão organizadora do Edital SENAI SESI de Inovação.

Definidas as partes interessadas nos projetos, a próxima etapa identifica os indicadores de desempenho ,considerados estratégicos para medição durante 0 processo de execução, os quais foram identificados por meio de entrevistas. Para facilitar a escolha pelos envolvidos, foi apresentada uma relação de indicadores coletados em diferentes literaturas. Cabe destacar ainda que a avaliação do desempenho do projeto não se limita apenas à fase de desenvolvimento (pós-aprovação), pois características identificadas na etapa de seleção também possuem relevância para organizações investidoras.

Como conclusão, cinco indicadores foram selecionados para este trabalho:

Complexidade Tecnológica: por este indicador, que possui forte relação com as etapas de seleção e desenvolvimento, avalia-se o escopo tecnológico da inovação proposta para ser desenvolvida, bem como sua capacidade de migrar para diferentes alternativas dentro de sua cadeia de valor. Projetos que possuam alta capacidade de migração tendem a possuir um baixo risco tecnológico.

Evolução Física: ainda que apresentado em métodos clássicos de gestão de projetos, a análise do cumprimento do cronograma físico do projeto foi identificado como um dos indicadores de desempenho de projetos de inovação tecnológica. Por este 
indicador, os stakeholders avaliam quanto o projeto evoluiu dentro do escopo e planejamento propostos.

Evolução Financeira: apesar de possuir correlação com a evolução física, este indicador oferece às partes envolvidas do projeto, informações adicionais sobre a capacidade de se adquirir os recursos necessários para desenvolvimento do projeto. Esta informação possui alta relevância, em especial, para a comissão organizadora do Edital SENAI SESI de Inovação, que gerencia, nacionalmente, os recursos aplicados nos projetos.

Processos de suporte à inovação: está relacionado com a capacidade da empresa e da equipe de gestão de projetos em oferecer agilidade nos processos de tomada de decisão presentes em diversos pontos do projeto. Neste indicador, são avaliados aspectos relacionados a sistema de gestão de projetos formalmente estabelecido, liderança, clareza de objetivos, sistema de feedback técnico e gerencial e procedimentos especiais para práticas de inovação.

Projeção Financeira: presente na maior parte da literatura e considerado como indicador de desempenho estratégico, a projeção financeira avalia a atratividade e os custos envolvidos para incorporação da tecnologia no processo produtivo da empresa e o retorno que tais investimentos resultarão. Neste indicador, são verificados aspectos relativos ao valor do investimento para produção, custo de produção, vantagens estratégicas, múltiplas aplicações e índices econômicos (TIR, VPL, ROI etc.).

De posse dos indicadores, teoricamente, nesta etapa, realiza-se a medição do desempenho dos projetos. Entretanto, cabe destacar que estes indicadores possuem diferentes graus de importância para cada projeto, uma vez que o Edital SENAI SESI de Inovação possui projetos de variados setores industriais, executados por empresas de diferentes portes. Assim, faz-se necessária uma etapa anterior à medição para definição dos pesos de cada indicador.

Para estabelecimento dos pesos, será utilizado o método da análise de pares proposto por VALLEJOS \& GOMES (2005). O método consiste em criar uma matriz de comparação par a par em que o tomador de decisão deve informar o indicador de maior importância. Para exemplificar esta etapa, será feita a análise do exemplo proposto na Tabela 1.
Tabela 1 - Exemplo de matriz de comparação.

\begin{tabular}{|l|c|c|c|c|c|}
\hline & Indicador 1 & Indicador 2 & Indicador 3 & Indicador 4 & Indicador 5 \\
\hline Indicador 1 & & & & & \\
\hline Indicador 2 & 1 & & & & \\
\hline Indicador 3 & 1 & 3 & & & \\
\hline Indicador 4 & 1 & 4 & 3 & & \\
\hline Indicador 5 & 1 & 2 & 3 & 4 & \\
\hline
\end{tabular}

Na Tabela 1, é possível estabelecer uma ordenação dos critérios, baseado no número de preferências de um critério a outro. Neste exemplo, o "Indicador 1" foi preferido 4 vezes, o "Indicador 3" obteve três ocorrências, o "Indicador 4" obteve duas, o "Indicador 2" apenas uma e o "Indicador 5" não foi preferido em nenhuma comparação. Contabilizadas as preferências entre os indicadores, é possível determinar o peso de cada um, utilizando a Equação (1) (os resultados serão aproximados para um número inteiro). $\mathrm{O}$ resultado da utilização da equação no exemplo é mostrado na Tabela 2.

$$
W_{i}=4 \cdot \frac{N_{i}-N_{\min }}{N_{\max }-N_{\min }}+1
$$

Após o estabelecimento dos pesos dos indicadores, inicia-se o processo de medição do desempenho dos projetos. Para tornar o processo mais objetivo, foi definida uma faixa para cada indicador, a qual representará o desempenho do projeto no momento da medição. As faixas de desempenho de cada indicador estão representadas na Tabela 3.

Tabela 2 - Cálculo do peso dos indicadores.

\begin{tabular}{|l|c|c|}
\hline & $\begin{array}{c}\text { Número de } \\
\text { ocorrências }\end{array}$ & Peso do indicador \\
\hline Indicador 1 & 4 & 5 \\
\hline Indicador 2 & 1 & 2 \\
\hline Indicador 3 & 3 & 4 \\
\hline Indicador 4 & 2 & 3 \\
\hline Indicador 5 & 0 & 1 \\
\hline
\end{tabular}


Tabela 3 - Faixas de medição dos indicadores de desempenho.

\begin{tabular}{|c|c|l|}
\hline Indicador & Desempenho & \multicolumn{1}{|c|}{ Faixa } \\
\hline \multirow{3}{*}{$\begin{array}{c}\text { Complexidade } \\
\text { Tecnológica }\end{array}$} & 4 & Excelente \\
\cline { 2 - 3 } & 3 & Bom \\
\cline { 2 - 3 } & 2 & Regular \\
\cline { 2 - 3 } Evolução Física & 1 & Ruim \\
\cline { 2 - 3 } & 3 & Desvio físico $<10 \%$ \\
\cline { 2 - 3 } & 2 & $10 \% \leq$ Desvio físico $<20 \%$ \\
\cline { 2 - 3 } & 1 & Desvio físico $\leq 30 \%$ \\
\hline \multirow{4}{*}{$\begin{array}{c}\text { Evolução } \\
\text { Financeira }\end{array}$} & 4 & Desvio financeiro $<10 \%$ \\
\cline { 2 - 3 } & 3 & $10 \% \leq$ Desvio financeiro $<20 \%$ \\
\cline { 2 - 3 } & 2 & $20 \% \leq$ Desvio financeiro $<30 \%$ \\
\hline \multirow{3}{*}{$\begin{array}{c}\text { Processos de } \\
\text { suporte à } \\
\text { inovação }\end{array}$} & 4 & Desvio financeiro $\leq 30 \%$ \\
\cline { 2 - 3 } & 3 & Muito Satisfatório \\
\cline { 2 - 3 } & 2 & Patisfatório \\
\cline { 2 - 3 } & 1 & Insatisfatório \\
\hline \multirow{3}{*}{$\begin{array}{c}\text { Projeção } \\
\text { Financeira }\end{array}$} & 4 & Muito Satisfatório \\
\cline { 2 - 3 } & 3 & Satisfatório \\
\cline { 2 - 3 } & 2 & Pouco Satisfatório \\
\cline { 2 - 3 } & 1 & Insatisfatório \\
\hline
\end{tabular}

Cabe ressaltar que a medição deve ser realizada por uma equipe de especialistas com conhecimento sobre os projetos pertencentes ao estudo, os quais possuem os requisitos técnicos necessários para definição das faixas de medição de cada projeto.

Com os pesos e a escala de medição, é possível determinar o valor máximo de cada projeto, utilizando a Equação 2.

$$
Z_{\operatorname{máx}}=\sum_{i=1}^{5} 4 . W_{i}
$$

Em que: $\mathbf{Z}_{\text {máx }}$ é o valor máximo da medição dos indicadores de desempenho de um projeto; Wi é o peso do indicador de desempenho "i".

De forma análoga ao cálculo da pontuação máxima, pode-se medir o desempenho do projeto em um determinado ponto de medição, que estabelece o valor absoluto medido pelo somatório dos produtos entre o valor medido e seu respectivo peso de todos os cinco critérios.

$$
Z=\sum_{i=1}^{5} M_{i} W_{i}
$$

Em que: $\mathbf{Z}$ é o valor da medição dos indicadores de desempenho de um projeto; Mi é a medição do indicador de desempenho i de um projeto.

Por fim, como o objetivo é permitir a comparabilidade entre os projetos de um portfólio, calcula-se o valor relativo do desempenho de cada projeto, dividindo o valor medido pelo valor máximo. Esta variável será denominada Índice de Desempenho Global (IDG) do projeto.

$$
I D G_{i}=\frac{Z}{Z_{\max }} \cdot 100 \%
$$

Em que: $I D G_{i}$ é o valor percentual do desempenho do projeto em um determinado ponto de medição.

\section{Avaliação dos Resultados}

Definido o método de aplicação da ferramenta de gestão de projetos, foi realizado um estudo de caso com uma amostragem de 10 projetos de inovação tecnológica pertencentes à edição de 2012 do Edital SENAI SESI de Inovação. Para avaliação dos indicadores, tanto no estabelecimento dos pesos, quanto na avaliação do desempenho atual, contou-se com a participação de especialistas da comissão organizadora do Edital de Inovação. O resultado desta aplicação é apresentado na Tabela 4, resguardando o sigilo de cada projeto e das respectivas empresas participantes.

Analisando os resultados obtidos pela aplicação do método, é possível observar quais são os projetos com alto desempenho e quais possuem baixo desempenho. A vantagem desta técnica é a possibilidade de comparação entre projetos de natureza tecnológica distintas e empresas de diferentes portes e setores industriais.

Além disso, a etapa de estabelecimento de pesos oferece à equipe de execução do projeto uma visão das prioridades avaliadas pelos stakeholders. Como exemplo de análise, observa-se que o Projeto 05 obteve pontuação absoluta (sem considerar os pesos) de 15 pontos e o Projeto 07 obteve 
Tabela 4 - Aplicação do método em 10 Processos de suporte à inovação tecnológica

\begin{tabular}{|c|c|c|c|c|c|c|c|}
\hline Projeto & $\begin{array}{l}\text { Mediçăo I } \\
\text { Peso }\end{array}$ & $\begin{array}{l}\text { Complexidade } \\
\text { Tecnológica }\end{array}$ & $\begin{array}{l}\text { Evoluçăa } \\
\text { Figica }\end{array}$ & $\begin{array}{l}\text { Evoluçäo } \\
\text { Financeira }\end{array}$ & $\begin{array}{l}\text { Gestäo da } \\
\text { Inovaçäo }\end{array}$ & $\begin{array}{l}\text { Projeçăa } \\
\text { Financeira }\end{array}$ & IDG \\
\hline \multirow{2}{*}{ Projeto 01} & Mediçâo & 3 & 4 & 2 & 4 & 3 & \multirow{2}{*}{$75,0 \%$} \\
\hline & Peso & 4 & 1 & 3 & 2 & 5 & \\
\hline \multirow{2}{*}{ Projeto 02} & Mediçấo & 2 & 3 & 1 & 2 & 3 & \multirow{2}{*}{$56,7 \%$} \\
\hline & Peso & 5 & 2 & 1 & 4 & 3 & \\
\hline \multirow{2}{*}{ Projeto 03} & Medicââ & 2 & 3 & 2 & 1 & 2 & \multirow{2}{*}{$46,7 \%$} \\
\hline & Peso & 4 & 1 & 2 & 3 & 5 & \\
\hline \multirow{2}{*}{ Projeto 04} & Medição & 4 & 3 & 1 & 3 & 2 & \multirow{2}{*}{$65,0 \%$} \\
\hline & Peso & 3 & 1 & 3 & 5 & 3 & \\
\hline \multirow{2}{*}{ Projeto 05} & Medição & 3 & 4 & 1 & 4 & 3 & \multirow{2}{*}{$70,0 \%$} \\
\hline & Peso & 2 & 4 & 5 & 3 & 1 & \\
\hline \multirow{2}{*}{ Projeto 06} & Medição & 2 & 2 & 1 & 3 & 3 & \multirow{2}{*}{$56,7 \%$} \\
\hline & Peso & 4 & 3 & 2 & 5 & 1 & \\
\hline \multirow{2}{*}{ Projeto 07} & Mediçấo & 3 & 3 & 2 & 2 & 4 & \multirow{2}{*}{$75,0 \%$} \\
\hline & Peso & 5 & 4 & 1 & 2 & 3 & \\
\hline \multirow{2}{*}{ Projeto as } & Medição & 1 & 4 & 2 & 2 & 4 & \multirow{2}{*}{$66,7 \%$} \\
\hline & Peso & 4 & 2 & 1 & 3 & 5 & \\
\hline \multirow{2}{*}{ Projeto 09} & Mediçâo & 4 & 4 & 3 & 3 & 3 & \multirow{2}{*}{$83,3 \%$} \\
\hline & Peso & 1 & 4 & 5 & 2 & 3 & \\
\hline \multirow{2}{*}{ Projeto 10} & Mediçấo & 3 & 3 & 1 & 3 & 2 & \multirow{2}{*}{$56,7 \%$} \\
\hline & Peso & 2 & 4 & 5 & 3 & 1 & \\
\hline
\end{tabular}

uma pontuação absoluta de 14 pontos. Contudo, quando se considera os pesos, a pontuação do Projeto 05 é de $70 \%$, enquanto o Projeto 07 é de $75 \%$, uma vez que as melhores notas do Projeto 07 foram nos critérios de maior peso, fato que não ocorreu no Projeto 05.

\section{Conclusões}

A aplicação do método apresentou alguns benefícios e limitações. O principal benefício é a possibilidade de comparação de desempenho de projetos de diferentes setores e indústrias de diferentes portes. Pela Tabela 4, é objetiva a análise e verificação do desempenho dos projetos, observando-se que o "Projeto 9" possui melhor desempenho e o "Projeto 3" o pior desempenho. Outro benefício do método é a visualização dos indicadores mais estratégicos para um determinado projeto. Pelo estabelecimento dos pesos, é possível, para a equipe executora, direcionar esforços nos indicadores de maior peso, priorizando ações que gerem maior impacto.

Existe ainda a possibilidade de se incorporar dinâmicas de jogos que propiciem uma competição entre as equipes dos projetos, incorporando mudanças comportamentais presentes nos jogos e, como consequência, estimulando os participantes a aumentarem seu desempenho. Como limitações do método, registra-se a característica estática da gestão, ou seja, o cálculo do desempenho do projeto é realizado em um determinado momento de verificação. Em nosso estudo de caso, a medição foi realizada em fevereiro/2014, a qual possivelmente é diferente do desempenho em maio/2014, por exemplo. Outra restrição encontrada é a inexistência de critérios de desempate entre projetos, fato que pode ocorrer com frequência quando se avalia uma grande quantidade de projetos. Por fim, conclui-se que o objetivo geral deste trabalho foi alcançado, dado ter sido apresentados métodos de gestão de projetos de inovação tecnológica que estimulem a participação dos stakeholders.

Com este trabalho, espera-se oferecer uma contribuição para os ambientes acadêmico e empresarial, podendo gerar benefícios que impactam em uma melhor utilização dos recursos do país e, consequentemente, e no crescimento econômico provocado pelo fomento às ações de inovação.

\section{Referências}

1. IBGE. (2013). Pesquisa de Inovaçao - PINTEC (p. 227). Rio de Janeiro. Retrieved from www.ipea.gov.br

2. Mason, C., \& Stark, M. (2004). What do Investors Look for in a Business Plan?: A Comparison of the Investment Criteria of Bankers, Venture Capitalists and Business Angels. International Small Business Journal, 22(3), 227-248.

3. Pillai, A. S., Joshi, a., \& Rao, K. S. (2002). Performance measurement of $R \& D$ projects in a multi-project, concurrent engineering environment. International Journal of Project Management, 20(2), 165-177.

4. PMBOK Guide. (2013). A Guide to the Project Management Body of Knowledge (5a Edition.).

5. Vallejos, R., \& Gomes, J. (2005). APPLYING A BENCHMARKING METHODOLOGY TO EMPOWER A VIRTUAL ORGANISATION. Sixth IFIP International Conference on Information Technology for Balanced Automation Systems in Manufacturing and Services, 279-286.

6. Zichermann, G., \& Cunningham, C. (2011). Gamification by Design. Oreilly \& Associates Inc. Retrieved from http:// medcontent.metapress.com/index/A65RM03P4874243N.pdf

\section{Mateus S. Freitas* \& Jefferson O. Gomes}

Departamento Nacional do Serviço Nacional de Aprendizagem Industrial.

*E-mail: msfreitas@cni.org.br 
